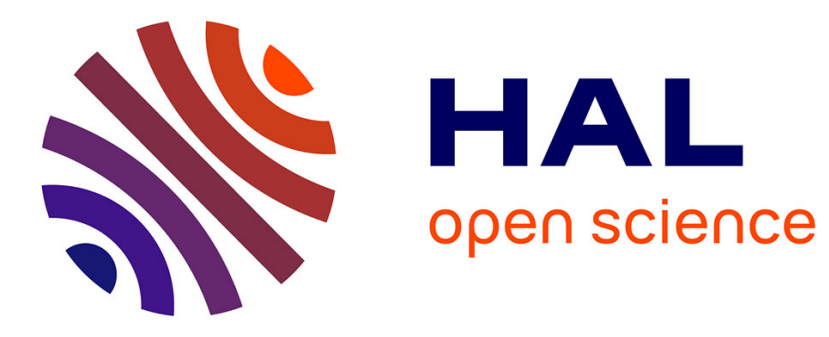

\title{
Logistique urbaine : refonder les logiques d'intermédiation
}

Odile Chanut, Gilles Paché, Falk Wagenhausen

\section{To cite this version:}

Odile Chanut, Gilles Paché, Falk Wagenhausen. Logistique urbaine : refonder les logiques d'intermédiation. Revue management \& avenir, 2012, 51 (1), pp.186-207. 10.3917/mav.051.0186 . hal-01767221

\section{HAL Id: hal-01767221 \\ https://hal.science/hal-01767221}

Submitted on 16 Apr 2018

HAL is a multi-disciplinary open access archive for the deposit and dissemination of scientific research documents, whether they are published or not. The documents may come from teaching and research institutions in France or abroad, or from public or private research centers.
L'archive ouverte pluridisciplinaire HAL, est destinée au dépôt et à la diffusion de documents scientifiques de niveau recherche, publiés ou non, émanant des établissements d'enseignement et de recherche français ou étrangers, des laboratoires publics ou privés. 


\title{
Logistique urbaine : refonder les logiques d'intermédiation
}

\author{
Odile Chanut $^{1}$, Gilles Paché ${ }^{2}$ et Falk Wagenhausen ${ }^{3}$
}

Les questions relatives à la logistique urbaine occupent désormais une place centrale dans la recherche en management. Longtemps confinée à des approches opérationnelles, notamment en termes d'organisation optimale des transports de marchandises dans des villes saturées, la logistique urbaine retient l'attention des chercheurs et des praticiens à partir de modèles stratégiques originaux, mettant l'accent sur l'importance d'une mutualisation des ressources entre entreprises concurrentes. La mutualisation apparaît essentielle à la fois sur un plan économique et sur un plan écologique, mais pose de redoutables problèmes de coordination pour le pilotage des flux. L'article aborde tout particulièrement la thématique de l'intermédiation en contexte de logistique urbaine en référence aux parties prenantes pouvant s'en charger, au premier rang desquelles figurent les prestataires de services logistiques et les grossistes.

Issues related to urban logistics have taken a central place in management research. Longtime reduced to operational approaches, particularly in terms of optimal organization of the transport of goods in saturated cities, urban logistics attracts the attention of academicians and practitioners taken from original strategic models, focusing on the importance of resource pooling among firms in competition. Pooling is essential both from an economic and an ecological point of view, but poses major problems in terms of flow monitoring. The article specifically addresses the topic of intermediation in the context of urban logistics, in reference to stakeholders who can do it, primarily among the logistics service providers and wholesalers.

«Qui est capable de conduire sans perdre son sang-froid à Marseille est capable de conduire dans le monde entier ! », dit-on souvent sur le ton de la galéjade méridionale. Pardelà la pointe d'humour se dissimule pourtant une réalité souvent peu agréable. Il suffit d'emprunter l'Autoroute Nord, qui débouche sur la ville, le matin d'une semaine ordinaire, puis de bifurquer vers les artères qui bordent la fameuse Canebière de Marcel Pagnol, pour découvrir un flot incessant de véhicules de livraison, prêts à s'arrêter brutalement, et sans vergogne, en double file pour décharger un colis dans une boutique de l'hyper-centre. Un entrelacs de petites ruelles, peu de larges boulevards, un trafic très chaotique, sans oublier une surprenante conduite à la marseillaise : tout est réuni pour faire prendre conscience au simple observateur ce que peuvent signifier, au concret, les contraintes de logistique urbaine, et l'importance d'une action publique pour éviter que les comportements anarchiques des uns et des autres finissent par totalement paralyser l'espace urbain, sans parler des impacts désastreux en termes de pollution de l'air.

\footnotetext{
${ }^{1}$ Maître de Conférences en Sciences de Gestion, CRET-LOG, Aix-Marseille Université. Courriel: odile.chanut@univ-amu.fr.

${ }^{2}$ Professeur des Universités en Sciences de Gestion, CRET-LOG, Aix-Marseille Université. Courriel : gilles.pache@univ-amu.fr.

${ }^{3}$ ATER en Sciences de Gestion, CRET-LOG, Université d'Avignon \& des Pays de Vaucluse. Courriel : wagenhausen@fast-special.com.
} 
Pendant longtemps, les embouteillages récurrents de Marseille, tout comme sa fantasque équipe de football, ont servi de toile de fond à un sympathique folklore local, d'ailleurs présent, à des degrés moindres, dans d'autres grandes métropoles d'Europe. Tout se passait alors comme si les espaces urbains encombrés étaient une inévitable malédiction liée à la métropolisation croissante de nos Sociétés. Force est de reconnaître qu'une page se tourne depuis moins d'une décennie. Il apparaît évident que la désorganisation en matière de livraisons génère des coûts jugés insupportables par de multiples parties prenantes, coûts qui sont d'ailleurs autant économiques qu'écologiques. La ville doit devenir « durable », c'est en tout cas le souhait de plus en plus de collectivités locales. Pour cela, il s'avère nécessaire de repenser les schémas de logistique urbaine à partir de logiques de coordination rigoureusement planifiées. Les chargeurs voulant pénétrer l'espace urbain vont notamment devoir accepter des contraintes collectives nouvelles, sans pouvoir agir de manière individuelle, comme ils l'ont fait jusqu'à présent.

Accompagnant les mutations en cours, un nouveau concept a émergé, celui de city logistics, qui correspond au processus d'optimisation globale des activités de transport et de logistique, initiées par des entreprises privées, dans les différentes zones urbaines, en tenant compte des contraintes de circulation, de consommation d'énergie et du respect de l'environnement des usagers (Taniguchi et Thompson, 2008). Une rapide consultation des bases de données académiques permet d'identifier plusieurs centaines de contributions sur le sujet, relatant des expériences de collectivités territoriales qui ont choisi de s'impliquer fortement dans le pilotage des opérations de logistique urbaine sur leur territoire, y compris en créant des fonctions de «managers de ville ». Leur objectif n'est pas de se substituer aux entreprises privées mais plutôt de les accompagner dans la formalisation de systèmes mutualisés, c'est-à-dire partagés entre elles, même lorsque ces entreprises sont en compétition frontale. Il s'agira, par exemple, d'imposer que des magasins d'enseignes de distribution concurrentes, localisés le long d'une même avenue, soient approvisionnés par des moyens logistiques communs, si possible en utilisant des véhicules hybrides moins polluants.

Cette réalité nouvelle interpelle autant les praticiens que les chercheurs. Amener des entreprises en compétition à travailler ensemble n'est pas chose aisée, surtout si l'une d'elles dispose d'un avantage concurrentiel en matière de logistique urbaine et rechigne, fort naturellement, à le partager avec d'autres. On retrouve là le débat connu sur les pratiques coopétitives, désormais d'une brûlante actualité en management stratégique (Dagnino et Rocco, 2011), et dont la city logistics offre un terrain d'investigation privilégié. L'angle d'attaque retenu dans l'article est complémentaire et pose une question simple : quel acteur tiers est apte à se positionner comme partenaire des collectivités locales pour assurer l'intermédiation entre la logistique massifiée, en amont des espaces urbains, et la logistique capillaire, au sein de ces mêmes espaces urbains ? La question apparaît essentielle, elle est pourtant encore peu explorée. Si de nombreuses parties prenantes semblent disposer de la légitimité et de l'expertise nécessaires, les expérimentations en cours ne permettent pas de dégager de véritable modèle à visée universaliste. Il serait donc présomptueux d'en tirer des conclusions hâtives sur le devenir des schémas de logistique urbaine. Plus modestement, nous posons la question du positionnement possible des prestataires de services logistiques (PSL), d'un côté, des grossistes, de l'autre, sur ce nouveau segment d'activité, en s'interrogeant sur lequel de ces deux membres du canal de distribution possède finalement la réelle compétence de management de la logistique capillaire ${ }^{4}$.

\footnotetext{
${ }^{4}$ Les auteurs remercient deux évaluateurs anonymes de Management \& Avenir pour leurs commentaires et stimulantes suggestions sur une première version de l'article.
} 


\section{Logistique urbaine : nouveaux enjeux, nouveaux acteurs, nouveaux schémas}

En l'espace d'une décennie, les questions liées à la gestion optimisée de la logistique urbaine ont donné lieu à une abondante littérature, entre autres sous la forme de conférences internationales et de numéros spéciaux de revues académiques. Nulle réelle surprise à un tel engouement car si elle a été longtemps confinée à des aspects purement opérationnels, l'organisation de tournées de livraison dans les centres villes, la logistique urbaine soulève désormais de nombreux enjeux économiques et sociétaux qui ne peuvent laisser indifférents les preneurs de décision publics et privés. Certains pays d'Europe, au premier rang desquels l'Allemagne, ont initié des réflexions de première importance sur l'amélioration de la circulation des marchandises dans la ville, sans doute parce que la sensibilité écologique, à travers le dynamisme du mouvement des Verts, y a été précoce. Mais quels sont les acteurs à coordonner afin d'éviter une multiplication anarchique d'initiatives individuelles ? Certains d'entre eux ont-ils pris une certaine avance en matière de pilotage des flux dans un contexte de logistique urbaine ? Ces interrogations appellent des réflexions afin de comprendre les enjeux actuels de l'intermédiation.

\subsection{Enjeux économiques et sociétaux}

Force est de constater que dans nos Sociétés occidentales caractérisées par un phénomène de surconsommation, le transport de marchandises en vue d'acheminer les produits finis jusqu'aux consommateurs finaux s'est considérablement intensifié. À titre d'illustration, le Ministère allemand en charge du transport estimait dès 2000 que le transport de marchandises connaîtrait dans le pays une hausse de $60 \%$ en 18 ans, entre 1997 et 2015 ; ses prévisions se sont avérées totalement fondées. La France est victime d'une évolution comparable : au carrefour des principales routes du Continent, elle supporte une grande partie du trafic intraeuropéen, et celui-ci a cru, entre 1990 et 2000, de $70 \%$ (ADEME, 2006). Le transport intranational de marchandises n'est pas en reste : il a augmenté de $30 \%$ en France, sur la même période. La destination finale des produits finis, acheminés de manière massive par transport routier, alors que le transport ferroviaire a décliné, est bien souvent l'espace urbain.

Plusieurs causes sont à l'origine du phénomène: un mouvement de métropolisation observé dans les économies occidentales depuis une centaine d'années, donnant naissance à des agglomérations importantes où se concentrent les consommateurs; des consommateurs qui, justement, sont de moins en moins prêts à se déplacer dans les hypermarchés en zones périurbaines pour faire leurs courses et redécouvrent les magasins de proximité ; de grands distributeurs alimentaires multipliant les nouveaux concepts de citymarkets; enfin, une hausse rapide du commerce électronique impliquant des livraisons au domicile des internautes. Ces nouveaux comportements de consommation induisent une augmentation importante des flux de marchandises en centre ville, avec leur lot de nuisances : la saturation des espaces urbains et la pollution, qu'elle soit mesurable en termes d'impact carbone ou en termes sonores. On doit hélas admettre que la situation devient critique, dégradant de manière significative la qualité de vie des urbains et l'image des villes (Juan, 2007).

Ainsi, dans certaines villes allemandes comme Düsseldorf ou Dortmund, les seuils maximaux annuels d'émission de particules, imposés par la directive européenne du $1^{\mathrm{er}}$ janvier 2005, sont désormais atteints aux premiers jours du printemps (Drewitz, 2007). Conscientes de la situation, des collectivités se sont engagées dans un projet de ville durable. Certaines envisagent d'instaurer des péages aux portes de la Cité, à l'image de Londres. D'autres lancent diverses initiatives susceptibles de diminuer de manière significative le stock de véhicules circulants. C'est le cas de Paris, ville «exemplaire » s'il en est (Ripert et 
Browne, 2009), et de son opération Autolib qui, sur le modèle du Vélib, met à disposition depuis décembre 2011 des automobiles électriques en libre-service et en «trace directe » (on peut prendre un véhicule à un point de la ville et le restituer dans un autre point, pour une somme modique et des formalités allégées). Le projet concernera, à l'horizon de la fin 2012, 46 communes de la région parisienne, gérant au total 1.120 stations.

Au-delà des expériences en cours, de nouvelles recherches sont attendues en matière de logistique urbaine pour penser et implanter des schémas de circulation entièrement renouvelés, adaptés à l'augmentation considérable des flux de marchandises en centre ville, et intégrant en outre l'objectif de développement durable. Ces nouveaux schémas, reposant sur des systèmes originaux de stockage, de transit et de transport (Císařová et Šroký, 2009), supposent de reconsidérer les relations inter-organisationnelles au sein des chaînes logistiques multi-acteurs pour mutualiser certaines ressources entre entreprises complémentaires et/ou concurrentes, et ainsi éviter de coûteuses redondances, en termes économiques et écologiques. En effet, de multiples organisations sont directement concernées dans l'élaboration des nouveaux schémas logistiques capables d'intégrer les contraintes de saturation d'espace et de développement durable. Il en résulte une gouvernance actuellement tâtonnante, ou «chemin faisant », qui laisse émerger des configurations propices à l'expérimentation et nous interroge sur le rôle que chaque acteur peut y jouer.

\subsection{Une grande diversité d'acteurs à coordonner}

Un très grand nombre d'acteurs sont parties prenantes de l'élaboration de nouveaux schémas logistiques pour l'acheminement des produits en centre ville, rendant complexes les reconfigurations envisageables (Russo et Comi, 2011). Deux cercles de parties prenantes peuvent être distingués: les acteurs directement impliqués dans les nouveaux schémas (parties prenantes principales); les acteurs supports des entreprises ou représentants des intérêts de tel ou tel public (parties prenantes subsidiaires). La Figure 1 en propose une synthèse dans le contexte français. Laisser chaque acteur agir en toute liberté sur le plan logistique va aboutir à une congestion des zones résidentielles avec une multiplication anarchique des véhicules de livraison, pas nécessairement optimisés concernant leur taux de remplissage ; les impacts environnementaux (émissions de $\mathrm{CO}_{2}$, embouteillages récurrents, etc.) et les dysfonctionnements économiques (incapacité à respecter les fenêtres de livraison exigées par les clients) en seront vite insupportables pour tous. Une combinaison harmonieuse d'initiatives privées et de politiques publiques apparaît donc indispensable pour développer un système durable de fret urbain. La city logistics intègre bien sûr, en premier lieu, les membres «classiques » du canal de distribution, qui constituent le premier cercle des parties prenantes :

- Il s'agit d'abord des inducteurs de flux, principalement des industriels et des distributeurs : la grande distribution alimentaire, mais aussi les réseaux sélectifs ou exclusifs, intégrés ou contractuels, pour une large part implantés en centre ville et dans les centres commerciaux; les pure players et autres opérateurs du commerce électronique; les industriels, comme les brasseurs qui livrent directement le circuit hors domicile; les grossistes, enfin, qui assument une fonction consistant à acheter à la production ou à l'importation des marchandises d'une façon continue, en quantités importantes, à les stocker, à y incorporer éventuellement du service, et à assurer l'approvisionnement régulier des utilisateurs professionnels (détaillants, collectivités, artisans, etc.) (Vandercammen et Jospin-Pernet, 2010).

- Il s'agit, ensuite, de gestionnaires de flux et/ou d'infrastructures, actuels ou à venir, à savoir les fournisseurs traditionnels de services logistiques (les PSL conventionnels, les messagers express), ainsi que les transporteurs de personnes (SNCF, RATP à Paris), qui disposent de structures logistiques pouvant être transformées en centres de distribution 
urbaine (CDU), telles que des gares désaffectées. On peut ajouter les acteurs supports de flux, tels que les fournisseurs d'énergie (EDF, GDF, AREVA) et autres fournisseurs de solutions «propres» pour le transport: constructeurs automobiles susceptibles de proposer des véhicules moins polluants en termes de $\mathrm{CO}_{2}$ comme en termes de nuisances sonores, mais aussi gestionnaires de parcs automobiles pouvant apporter des solutions d'optimisation dans l'utilisation des véhicules en circulation.

- Il s'agit, enfin, de régulateurs de flux, les villes et collectivités territoriales, ayant un rôle plus politique dans la gestion des marchandises dans l'espace urbain. Traditionnellement en charge des questions d'urbanisme, elles ont jusque là laissé les distributeurs inducteurs de flux développer des structures et schémas logistiques de manière anarchique, chaque système d'offre déployant ses propres ressources, de manière autonome. Les villes et collectivités territoriales se sont récemment emparées de la question et elles pourraient se positionner en tant que pivot de la coordination. Au-delà d'un simple pouvoir coercitif, en réglementant par exemple de façon drastique les livraisons en centre ville, les collectivités territoriales sont aussi aptes à impulser une action concertée avec les entreprises dans un objectif de mutualisation horizontale des ressources logistiques entre systèmes d'offre concurrents, qu'il s'agisse de réseaux de franchise ou d'enseignes de distribution alimentaire, à travers leurs multiples magasins de proximité.

En complément des acteurs directs du premier cercle, un second cercle de parties prenantes doit être introduit; il dispose, lui aussi, d'un pouvoir d'influence au sens de Milgrom et Roberts (1990). On y trouve des syndicats, des fédérations professionnelles et des chambres de commerce et d'artisanat. Associations supports de firmes, elles représentent directement les intérêts de leurs membres; au-delà, elles leurs apportent de multiples services en termes d'informations et de conseils, de formations et de concertation. Elles peuvent jouer un rôle précieux dans la coordination de leurs membres pour dégager des solutions innovantes et emporter l'adhésion. La Fédération Française de la Franchise (FFF) fait ainsi partie de l'environnement institutionnel des réseaux contractuels, capable d'initier des réflexions sur la question du développement durable ou celle de la mutualisation de ressources entre réseaux concurrents. De la même manière, la Confédération Française du Commerce Inter-entreprises, Commerce de Gros, Commerce International (CGI), organisation professionnelle représentative de l'ensemble du négoce, commerce de gros et commerce international, représente, défend et informe ses adhérents sur les grands enjeux de la branche ; à ce titre, elle peut faciliter la concertation de ses fédérations sur les nouveaux schémas de logistique urbaine.

Mais le second cercle de parties prenantes va beaucoup plus loin. Il intègre des associations de riverains et associations de consommateurs, qui représentent, à l'autre bout de la chaîne logistique multi-acteurs, les usagers de l'espace urbain et les bénéficiaires des livraisons de marchandises. Enfin, les organismes de recherche, publics ou privés, sont parties prenantes grâce à leur effort de financement des innovations, tant techniques qu'organisationnelles. Nous citerons, à titre d'exemple, l'Agence de l'Environnement et de la Maîtrise de l'Énergie (ADEME), établissement public à caractère industriel et commercial placé sous la tutelle conjointe des Ministères en charge de l'Écologie, du Développement Durable, des Transports et du Logement, de l'Enseignement Supérieur et de la Recherche, et de l'Économie, des Finances et de l'Industrie. Sa mission est de participer à la mise en œuvre des politiques publiques dans les domaines de l'environnement, de l'énergie et du développement durable. Pour ce faire, elle met à disposition des entreprises, des collectivités locales, des Pouvoirs publics et du grand public, ses capacités d'expertise et de conseil. Elle aide, en outre, au financement de projets, à la recherche et à la mise en œuvre et cela, dans différents domaines dont la gestion des déchets, la préservation des sols, l'efficacité énergétique et les énergies renouvelables, la qualité de l'air et la lutte contre le bruit et le 
développement de villes et territoires durables. Elle est en conséquence une ressource clé pour les questions relatives à la logistique urbaine.

Au final, le nombre et la diversité des parties prenantes rendent complexe la recherche de solutions de synthèse et la coordination des acteurs pour faire tendre leurs efforts dans une même direction : une ville durable avec des flux de marchandises contrôlés (Quak, 2011). La démarche oblige aussi à reconsidérer les critères de performance de la logistique en milieu urbain : aux critères économiques (coût, délai, réactivité) doivent être ajoutés des critères sociétaux tels que la réduction des nuisances de toutes natures (bruit, pollution, saturation de l'espace urbain, redondance des moyens). On retrouve ici, de façon indirecte, la lancinante question de la mesure de la performance dans les structures en réseau, soulignée par Frédouet et Le Mestre (2005) : "Le réseau cherche à atteindre un double objectif de performances particulières et de performance collective. Le suivi des performances ne peut alors se réduire à la juxtaposition des suivis réalisés par chacun des membres. Dans la mesure où des objectifs sont assignés spécifiquement au réseau, celui-ci doit en évaluer le degré de réalisation. Il est alors nécessaire de définir un système d'évaluation global regroupant un ensemble de critères financiers et non financiers ». Les enjeux de l'intégration coordonnée des structures logistiques en contexte urbain s'analysent, de façon concomitante, en termes d'efficience économique, de partage des bénéfices de la coopération et de gouvernance, par l'un des acteurs, des opérations liées à la city logistics. Dès l'instant, l'une des questions centrales qui se pose au chercheur est le rôle possible que pourraient tenir, dans ces nouveaux schémas, les différents membres historiques du canal de distribution, entre autres les grossistes et les PSL. 
Figure 1 : De nombreuses parties prenantes à coordonner pour les nouveaux schémas de la logistique urbaine ${ }^{5}$

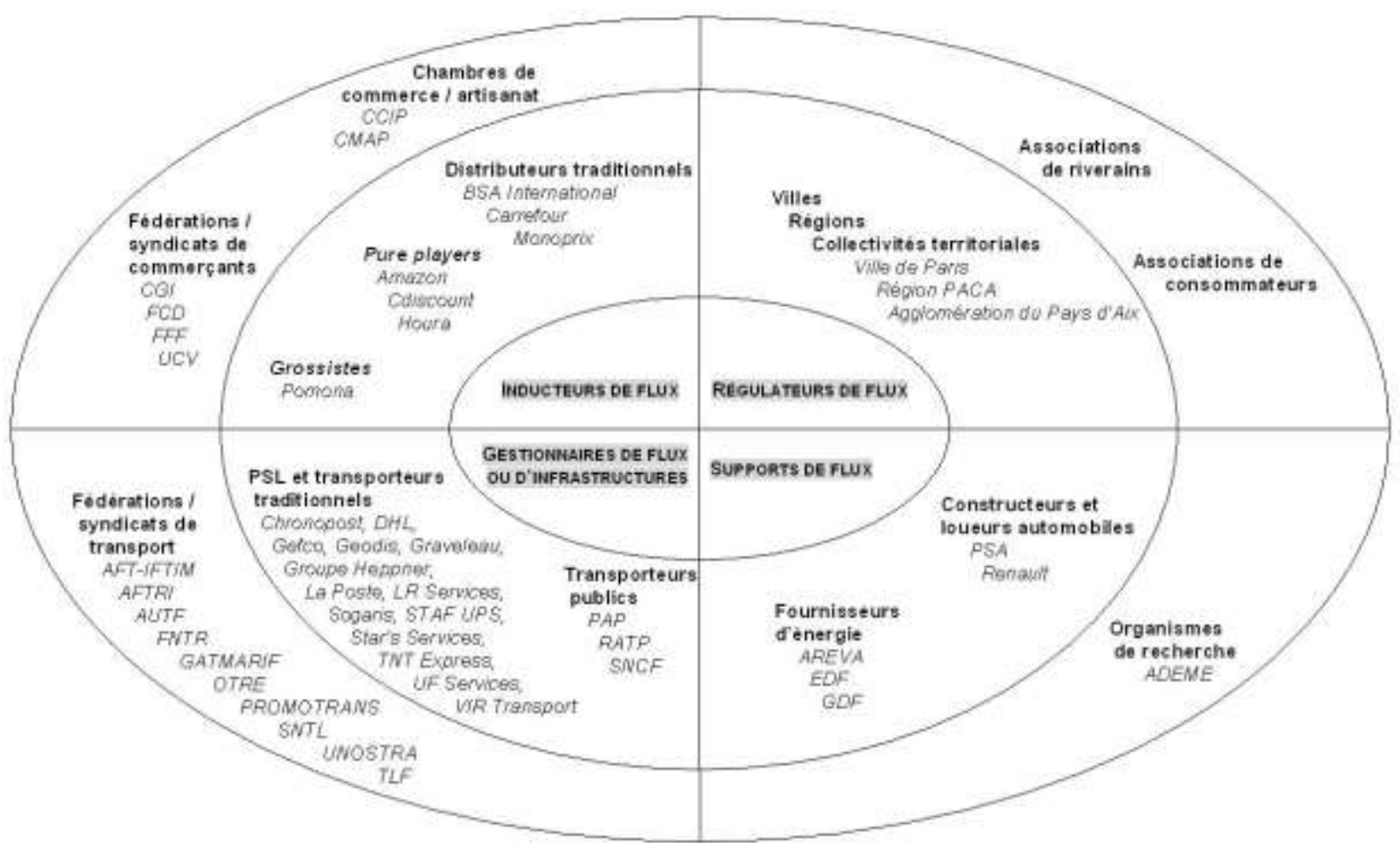

\subsection{Vers l'émergence de nouveaux schémas logistiques}

Deux directions complémentaires sont actuellement empruntées en matière de logistique urbaine : d'une part, la mutualisation plus ou moins systématique de ressources logistiques, d'autre part, la combinaison harmonieuse de moyens de transport alternatifs. La mutualisation se présente comme une manœuvre collective incontournable, au service d'objectifs économiques et sociétaux (Chanut et Paché, 2012). Elle consiste à la mise en commun, par des entreprises distinctes (et parfois concurrentes), de ressources logistiques telles que des entrepôts ou plates-formes, des équipements de manutention et des véhicules, mais aussi des ressources humaines, telles que les chauffeurs-livreurs ou manutentionnaires. Les expériences de mutualisation peuvent être qualifiées de verticales lorsqu'elles aboutissent à la mise en commun de moyens entre entreprises situées à des niveaux différents du canal de distribution, par exemple entre un industriel et un distributeur. Elles peuvent être qualifiées d'horizontales lorsqu'elles impliquent des entreprises ou groupes d'entreprises directement concurrentes au même stade de la chaîne logistique multi-acteurs, par exemple entre deux enseignes de distribution concurrentes. Certains inducteurs de flux ont d'ores et déjà développé une réelle expertise en termes de logistique de livraison de marchandises dans la ville et maîtrisent parfaitement la mutualisation verticale. C'est le cas de la grande distribution alimentaire ou des réseaux de franchise, qui mutualisent depuis les années 1990, selon un modèle vertical, les ressources logistiques destinés à l'approvisionnement de l'ensemble de leurs magasins, et qui expérimentent depuis peu une mutualisation horizontale (Chanut et al., 2011).

\footnotetext{
${ }^{5}$ Il est à noter que certains des acteurs endossent plusieurs rôles. Par exemple, le distributeur Monoprix, classé dans les inducteurs de flux (distributeur traditionnel) dispose de sa propre flotte de camions; il est donc aussi gestionnaire de flux. Les grossistes sont inducteurs de flux lorsqu'ils sont négociants, et simplement gestionnaires de flux lorsqu'ils sont courtiers. Nous avons choisi de positionner les acteurs dans la Figure par rapport au rôle principalement joué.
} 
Au niveau de la ville, la mutualisation horizontale peut prendre la forme de CDU dont l'objet est la massification des flux et l'optimisation des tournées à destination des commerçants et/ou industriels d'une zone urbaine. Il s'agit ici de diminuer le nombre de trajets de camions ou camionnettes, et souvent de créer des possibilités d'utilisation de matériels plus «urbains » (et moins polluants) pour réduire les incidences du transport de marchandises sur les niveaux de pollution et sur l'occupation de l'espace public, tout en améliorant l'efficacité de la distribution pour les transporteurs et les destinataires. Au plan fonctionnel, une telle organisation se traduit souvent par le regroupement, sur une plate-forme unique, des produits à destination de la zone à livrer, ce qui génère une rupture de charge. On voit également apparaître de nouveaux services innovants qui cherchent à valoriser la ville et ses activités en substituant des flux de produits gérés « industriellement » à des déplacements en voiture plus ou moins anarchiques car organisés individuellement. Compte tenu de l'encombrement dont les livraisons à domicile sont à l'origine, certains transporteurs spécialisés en messagerie et quelques PSL commencent à proposer des alternatives séduisantes : des stations et points relais livraison (avec livraison gratuite pour inciter les clients à les préférer), ou encore des consignes logistiques urbaines (Durand et Senkel, 2011), dont l'un des exemples les plus connus est la packstation, une sorte de «boîte aux lettres » pour produits, localisée dans les lieux de grand passage (gares, stations de métro). Amazon.com, la célèbre librairie en ligne, travaille sur ce type de système depuis 2009.

D'autres initiatives impliquant plusieurs parties prenantes sont aujourd'hui prises en matière d'acheminement des produits. Elles consistent à utiliser les infrastructures de transport qui traversent la ville pour plusieurs usages alternatifs (transport de voyageurs, transport de marchandises), à l'instar de l'expérience menée à Dresde par le constructeur automobile Volkswagen et l'entreprise en charge du transport de voyageurs, la DVB AG (voir l'encadré 1). Ces nouveaux concepts logistiques, désignés par le vocable TramFret, retiennent l'attention et pourraient largement se généraliser au-delà des frontières de l'Allemagne (Drewitz, 2007). L'exemple du partenariat entre le distributeur Monoprix et la SNCF, à Paris, en témoigne de manière éclatante ; l'acheminement des produits dans la Capitale en direction des différents magasins Monoprix devrait être effectué, à moyen terme, par le biais des voies ferrées existantes. Et d'autres exploitants de transport en commun en France, comme la RATP, commencent à s'intéresser sérieusement à ces solutions pour le moins originales (Augereau, 2009 ; PIPAME, 2009). Il faut cependant prendre garde aux limites juridiques des politiques d'accès restrictif au centre ville, comme en témoignent les différents recours en justice de plusieurs transporteurs contre les autorités de la ville de Vicence, en Italie (Dablanc et al., 2010).

Encadré 1 : L'expérience de TramFret de Dresde (Allemagne)

À la fin des années 1990, Volkswagen, lors de la création d'une nouvelle usine produisant une voiture de prestige, s'est donné comme objectif de communication de rendre les activités de production visibles de l'extérieur (une «manufacture en verre »). Pour ce faire, il fallait trouver un emplacement adéquat. Le choix se porta sur des locaux disponibles à proximité du centre historique de Dresde. Mais encore fallait-il trouver un moyen d'acheminer les matériaux nécessaires vers l'usine en centre ville, compatible avec les exigences écologiques des habitants de Dresde et de ses élus. La solution, innovante consista à mettre en circulation un TramFret, appelé CarGoTram, qui utilise les voies du tramway destiné au transport de personnes exploitées par DVB AG. Mis en service le $1^{\text {er }}$ mars 2001, le CarGoTram est géré par le centre de commandement de la DVB, sans gêner la circulation des tramways qui transportent les passagers. Il achemine à travers le centre ville historique tous les composants nécessaires à la construction des véhicules automobiles, sauf la carrosserie, et cela 6 jours sur 7, 21 h sur 24, selon une logique de juste à temps. Chaque TramFret, d'une longueur de 60 mètres, peut charger jusqu'à $214 \mathrm{~m}^{3}$ ou bien 60 tonnes de matériaux. Une tournée de TramFret représente l'équivalent de trois poids-lourds. Le CarGoTram participe ainsi à 
l'amélioration de la qualité de vie et augmente l'attractivité de la ville. Il suppose toutefois une parfaite coordination des opérations entre le constructeur automobile et l'exploitant de transport de voyageurs de la ville.

Source : d'après http://www.dvb.de/de/Die-DVB-AG/Zahlen-Daten/CarGoTram/, consulté le 10 janvier 2012.

\section{Quel intermédiaire pour la logistique urbaine de demain ?}

Si la quête effrénée d'une logistique urbaine plus performante ne fait réellement plus de doute, compte tenu des enjeux économiques et sociaux en présence, il est important de s'interroger sur les acteurs pouvant initier, de par leur expertise singulière, des schémas innovants capables de répondre aux défis de demain. Une brève mise en perspective historique semble indiquer que les PSL, dont la présence sur le marché n'a cessé de croître depuis vingt ans (Fulconis et al., 2011), pourraient trouver ici une possible application de leur savoir-faire ancestral en termes de mutualisation. Sans sous-estimer l'expertise des PSL en la matière, force est néanmoins de reconnaître que les grossistes disposent de ressources et compétences distinctives que l'on pourrait imaginer aisément mobilisables en contexte de logistique urbaine. C'est notamment le cas des connaissances tacites accumulées par leurs chauffeurs-livreurs. Plus que jamais, la logistique urbaine a besoin de s'appuyer sur un ou des acteurs pivots capables d'initier une vision intégrative pour réduire les ruptures de chaîne (Friesz et al., 2011).

\subsection{L'apprentissage de la mutualisation verticale par le PSL}

Il est traditionnellement admis que les stratégies de recentrage des industriels puis des distributeurs sur quelques compétences centrales les ont conduits à se désengager d'activités jugées périphériques, au premier rang desquelles les activités logistiques. Les PSL, acteurs spécialisés dans la maîtrise des flux physiques et d'information associés, se sont développés en occupant un terrain nouveau : l'exploitation des opérations logistiques pour le compte d'autrui. Selon une étude déjà ancienne menée par le cabinet Eurosiris, les PSL ont trois origines en France : 1) les prestataires issus du transport comme les transporteurs routiers, les commissionnaires et les transitaires (organisateurs des chaînes de transport à l'international) et les integrators (messagerie express, transport aérien);2) les filiales de grands groupes manufacturiers, tels que Gefco (filiale logistique du groupe PSA), ou de grands groupes de distribution, tels que Easydis (filiale logistique du groupe Casino); et 3) les prestataires de services aux industries qui œuvrent généralement pour des secteurs de haute technologie en assurant des activités de manutention, d'entreposage voire d'assemblage terminal, tels que Daher. La fin des années 1980 marque l'essor considérable de l'industrie de la prestation logistique : les PSL se spécialisent alors peu à peu sur des marchés variés, et se dotent de puissantes infrastructures d'entreposage (entrepôts) et/ou de transit (plates-formes).

Un PSL ne peut plus s'apparenter uniquement à une entreprise mettant à la disposition de ses clients une activité d'acheminement des produits entre deux points de l'espace, même si la manœuvre transport reste bien un «service» de nature logistique dont le niveau de performance conditionne directement la performance d'une chaîne logistique multi-acteurs. Ainsi, les transporteurs express positionnés sur le marché de la livraison rapide avec des délais garantis, comme UPS, Fedex ou DHL Worldwide Express, commercialisent une prestation très recherchée dans de nombreux secteurs d'activité pour lesquels la rupture d'approvisionnement peut avoir des effets dramatiques (industries lourdes ou prêt-à-porter haut de gamme par exemple). Les PSL élargissent en fait leur système d'offre en proposant de 
nouveaux services aux clients dans le cadre d'un package associant transport, gestion des stocks, préparation des commandes, voire assemblage terminal des produits (Fulconis et al., 2011). L'élargissement du système d'offre permet ainsi au PSL de devenir un acteur incontournable de la chaîne logistique multi-acteurs, au risque de le voir s'y enraciner, avec les effets de dépendance de la part des chargeurs que l'on peut imaginer.

Pour des raisons historiques, la France est l'un des pays les plus dynamiques en matière de prestation logistique. Les PSL y ont construit des capacités d'entreposage de très grande taille et, en parallèle, ils ont également développé une expertise qui leur permet de mieux répondre aux besoins de leurs clients en proposant des solutions compétitives en matière de pilotage et de régulation des flux. La force des PSL est d'avoir très vite compris qu'en se situant à l'interconnexion de plusieurs chaînes logistiques multi-acteurs, par exemple en gérant des entrepôts communs à plusieurs distributeurs, ils s'avèrent capables de mutualiser des ressources en faisant ainsi jouer un double phénomène d'économies d'échelle et de champ. Incontestablement, les PSL tiennent une place de choix dans les mutualisations verticales et, plus récemment, horizontales. D'un point de vue historique, leur savoir-faire s'est néanmoins déployé de manière privilégiée le long des chaînes logistiques multi-acteurs, en associant plusieurs magasins autour d'infrastructures logistiques communes. L'approvisionnement en flux tendu des hyper et supermarchés sur le modèle du groupage destinataire dès les années 1980 est une excellente illustration de cette évolution.

Les PSL ont ainsi pu démontrer leurs capacités à impulser des logiques de massification des flux de plus en plus forte sur quelques nœuds logistiques (entrepôts et/ou plates-formes), judicieusement localisés dans l'espace, au barycentre des magasins à desservir (Guillo, 2008), pour bénéficier d'un prix du sol très attractif. Depuis les années 2000, de nouveaux schémas logistiques d'approvisionnement se sont multipliés dans le secteur de la distribution alimentaire. Ils reposent sur un système original fondé sur deux séquences connectées : dans un premier temps, les industriels livrent un entrepôt multi-fournisseurs (ou entrepôt primaire) au sein duquel les flux sont massifiés; dans un second temps, les industriels livrent - à plusieurs - les entrepôts de distributeurs (ou entrepôts secondaires) en fonction de leurs besoins, entrepôts qui livreront ensuite les magasins. Au sein de ces nouveaux schémas, il est possible de distinguer deux démarches différentes mais complémentaires : les pools et les centres de collaboration et de consolidation (CCC). Dans le cadre des pools (une dizaine en France, composés de deux à quatre fournisseurs), la mise en place d'entrepôts de massification reste à l'initiative d'industriels parfois concurrents, qui décident de regrouper leurs livraisons au sein d'un même entrepôt pour mutualiser le transport, le stockage, voire la gestion des approvisionnements en amont de l'entrepôt du distributeur. C'est le cas, par exemple, de Pepsi-Cola et d'Orangina, qui ont développé un co-camionnage de ramassage auprès des centres d'embouteillage, à destination des entrepôts du distributeur Auchan. Les industriels impliqués dans des logiques de mutualisation procèdent soit à un pooling industriel, soit à une gestion mutualisée des approvisionnements.

Dans le cadre des CCC, le transport amont entre les usines et le CCC est à la charge des industriels, tandis que le distributeur s'occupe de son côté des commandes mutualisées de $x$ industriels pour livrer ses différentes plates-formes. Les CCC s'inscrivent dans une démarche très industrialisée dont les avantages immédiats sont de plusieurs ordres: diminution de l'immobilisation financière chez le distributeur, amélioration de la disponibilité des produits et donc du chiffre d'affaires pour l'industriel, optimisation du taux de remplissage du transport amont. Le respect des contraintes environnementales n'est également pas à négliger, il correspond d'ailleurs à un critère de choix des PSL par les chargeurs de plus en plus saillant (Wolf et Seuring, 2010). Les stratégies actuelles de mutualisation des ressources logistiques conduisent ainsi à repenser les processus clé de la chaîne logistique multi-acteurs et l'affectation des ressources entre entreprises géographiquement distantes. Les 
PSL ont su astucieusement développer un système d'offre performant pour répondre aux besoins de leurs clients de la grande distribution alimentaire. Est-il adapté réellement aux contraintes de la logistique urbaine ? Rien n'est moins sûr, car l'expertise des PSL s'est construite sur le transport interurbain et international, et non pas sur la connaissance fine de l'espace urbain proprement dit.

\subsection{L'expertise des grossistes : quo vadis?}

Ayant longtemps occupé une place importante dans les canaux de distribution de produits industriels et de grande consommation, et même acteur majeur dans leur structuration (Jeanmougin-Lurdos, 1995), les grossistes ont en partie perdu de leur superbe. Le poids du commerce de gros alimentaire dans le produit intérieur brut a ainsi diminué régulièrement au cours des trente dernières années, notamment en France. Les raisons en sont connues. Elles sont liées aux mutations intervenues dans le circuit de distribution des produits alimentaires, avec la concentration des groupes de distribution et le rôle croissant joué par les centrales et groupements d'achat du commerce intégré et associé (Filser et al., 2012). L'avenir se serait-il assombri au point de n'offrir aucune opportunité de rebond aux grossistes ? Il y a plus de dix ans de cela, Dugot (2000) précisait que les grossistes pouvaient justifier leur utilité « en se positionnant par rapport à trois facteurs principaux : un certain type de clientèle, un certain type de produit et un territoire spécifique ». Une stratégie d'autant plus aisée à conduire, selon lui, que les entreprises du commerce de gros sont souvent des petites structures avec des zones de chalandise assez limitées ; leur avantage compétitif s'analyse en termes de proximité avec la clientèle et de connaissance du terrain : " proches de leur marché, elles apparaissent comme particulièrement indiquées pour l'approvisionnement de la petite clientèle et le traitement des commandes en petits lots » (Dugot, 2000). Par leur riche expérience, les grossistes restent ainsi très présents dans un certain nombre de canaux de distribution ayant un puissant ancrage urbain, au premier rang desquels la commercialisation des produits frais et des produits pharmaceutiques, et la restauration hors foyer; dans une ville comme Bologne, un tiers des livraisons vers les unités commerciales sont assurées par des grossistes (Danielis et al., 2010).

Mais de quoi parle-t-on exactement en matière d'intermédiation grossiste, en comparaison avec l'intermédiation dont les PSL sont à l'initiative ? À l'interface entre vendeurs et acheteurs, le grossiste gère en fait huit flux liés au produit, au transfert de titre de propriété, à la promotion, à la négociation, au financement, à la prise de risque, à la commande et au paiement (Rosenbloom et Larsen Andras, 2008); la Figure 2 en propose une synthèse en référence aux fonctions exercées. Filser (1989) distingue deux catégories de grossiste. La première catégorie est celle des négociants, propriétaires des marchandises et qui, de ce fait, supportent un maximum de risque ; ces grossistes se rémunèrent grâce à la marge prélevée. La deuxième catégorie est celle des courtiers, non-propriétaires des marchandises; ils se rémunèrent par une commission, c'est-à-dire un pourcentage sur le montant de la transaction effectuée entre deux acteurs. Par-delà la distinction juridique en termes de transfert de titres de propriété, Filser (1989) propose subtilement de caractériser les grossistes en fonction des trois principaux types de services procurés :

- Le premier type de service est de nature logistique, avec par exemple le stockage et le transport des marchandises, l'allotissement et la mise en rayon en magasin.

- Le deuxième type de service est de nature financière, avec par exemple la reprise des invendus, le crédit accordé au client et le financement du fournisseur.

- Le troisième type de service est de nature commerciale, avec par exemple la constitution d'assortiments, la recherche de débouchés pour le fournisseur et le fractionnement des quantités achetées par le client. 
Figure 2 : Les fonctions exercées par un grossiste

\section{Achat, transport, fractionnement, assortiment, allotissement}

stockage, mise a disposition et présentation, vente

Triptyque fonctionnel : achat / stockage / vente

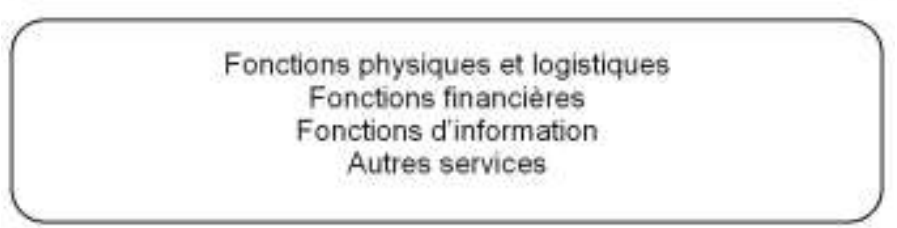

Source : d'après Dugot (2000).

Comme le note Filser (1989), lorsque plusieurs grossistes sont en concurrence, «ils peuvent chercher à se différencier en réduisant leurs coûts puis leurs prix. Un moyen de réaliser cette réduction passe par l'élimination de certains services auxquels un segment de clients est prêt à renoncer $»$. C'est le cas du cash \& carry, qui voit certains clients renoncer à une partie du service logistique, à savoir le transport terminal de mise à disposition des produits en magasin. Dans la mesure où nous souhaitons aborder le rôle que pourraient tenir les grossistes dans les schémas de logistique urbaine, il est naturel de se focaliser sur ceux qui sont impliqués dans le transport terminal. En effet, la question est de savoir si l'expertise accumulée en matière de gestion des tournées de livraison, pour une pluralité d'expéditeurs et de destinataires, pourrait être porteuse de pratiques de mutualisation performantes. Il est vrai qu'en associant dans un même véhicule des familles de produits logistiquement compatibles, et à destination des différents magasins d'un même quartier, les grossistes se sont placés par le passé comme des acteurs incontournables du transport des marchandises dans la ville.

Se fondant sur une analyse typologique par classification automatique, Cancelier (1981) a ainsi proposé une typologie des entreprises du commerce de gros en neuf classes. Chaque classe a en commun certaines caractéristiques explicatives permettant de discriminer les grossistes entre eux. L'auteur identifie tout particulièrement une classe dénommée grossistes dont la fonction de transport-livraison est privilégiée, qui représente presque un cinquième des entreprises. Dans cette classe, la majorité des entreprises reste de petite taille, avec un puissant ancrage régional. Leur activité commerciale est à destination des utilisateurs professionnels et elle se limite au marché intérieur. En d'autres termes, le grossiste orienté logistique témoigne d'un fort encastrement dans le tissu économique local, son expertise majeure repose sur la valorisation de logiques de proximité géographique et relationnelle avec la clientèle. Ceci est sans doute moins le cas des PSL, ayant développé des schémas logistiques fondés sur la massification des flux et la maîtrise du transport interurbain et international (voir le Tableau 1). En d'autres termes, le grossiste performant «est celui qui optimise la gestion de ses stocks et les contraintes temporelles de son système de livraison (...). Il correspond à une filière organisée avant tout en fonction d'impératifs logistiques, où le produit est valorisé par ses qualités de présentation et de conservation (résistance au transport en particulier) » (de Raymond, 2010). La question est de savoir si une telle expertise est apte à faire jouer un effet de levier dans une stratégie de positionnement sur le marché de la logistique urbaine. L'accent sera tout particulièrement mis sur les compétences du chauffeurlivreur. 
Tableau 1 : Éléments de comparaison entre le PSL et le grossiste

\begin{tabular}{|c|c|c|}
\hline & $P S L$ & Grossiste \\
\hline Définitions & $\begin{array}{l}\text { Entreprise prestataire de services assurant } \\
\text { la réalisation d'activités logistiques pour } \\
\text { le compte d'un industriel ou d'un } \\
\text { distributeur, dénommé chargeur }\end{array}$ & $\begin{array}{l}\text { Entreprise négociante qui achète des } \\
\text { produits pour revente à des clients (elle } \\
\text { assume un risque commercial) } \\
\text { Peut aussi être simple courtier (elle assure } \\
\text { alors une prestation logistique) }\end{array}$ \\
\hline $\begin{array}{l}\text { Clients } \\
\text { principaux }\end{array}$ & $\begin{array}{l}\text { Entreprises industrielles ou de distribution } \\
\text { ayant fait le choix de déléguer tout ou } \\
\text { partie de leurs activités logistiques pour se } \\
\text { recentrer sur leurs compétences clé }\end{array}$ & $\begin{array}{l}\text { Clients professionnels (détaillants, } \\
\text { collectivités, artisans, etc.), le plus } \\
\text { souvent de taille régionale ou locale }\end{array}$ \\
\hline $\begin{array}{l}\text { Horizon spatial } \\
\text { principal }\end{array}$ & Transport interurbain et international & Transport urbain \\
\hline $\begin{array}{l}\text { Structure de } \\
\text { marché et taille }\end{array}$ & $\begin{array}{l}\text { Marché quasi-oligopolistique sur lequel } \\
\text { les cinq premiers PSL détiennent un tiers } \\
\text { du marché en France }\end{array}$ & $\begin{array}{l}\text { Marché atomisé, même si on doit noter la } \\
\text { présence de grandes entreprises comme } \\
\text { Pomona, France Boissons ou Orexad }\end{array}$ \\
\hline Fonctions & $\begin{array}{l}\text { Au-delà de la simple maîtrise des activités } \\
\text { logistiques (préparation des commandes, } \\
\text { entreposage, transport), élargissement de } \\
\text { l'offre à des services à plus forte valeur } \\
\text { ajoutée, comme l'assemblage de } \\
\text { composants sur plates-formes modulaires, } \\
\text { la gestion du service après-vente ou des } \\
\text { centres d'appel des clients, etc. }\end{array}$ & $\begin{array}{l}\text { Achat pour revente, stockage et transport } \\
\text { des marchandises, avec incorporation } \\
\text { éventuelle de services commerciaux } \\
\text { (conseil pour l'assortiment, allotissement, } \\
\text { mise en rayon, promotions) ou financiers } \\
\text { (délais de paiement, crédit, reprise } \\
\text { d'invendus) }\end{array}$ \\
\hline $\begin{array}{l}\text { Compétences } \\
\text { distinctives }\end{array}$ & $\begin{array}{l}\text { Savoir-faire organisationnel : évolution } \\
\text { vers la maîtrise de conception des } \\
\text { systèmes logistiques }\end{array}$ & $\begin{array}{l}\text { Fine connaissance tacite de l'espace } \\
\text { urbain, et maîtrise de la capillarité }\end{array}$ \\
\hline $\begin{array}{l}\text { Facteurs clé de } \\
\text { succès }\end{array}$ & $\begin{array}{l}\text { Massification des flux sur entrepôts et/ou } \\
\text { plates-formes }\end{array}$ & $\begin{array}{l}\text { Connaissances spatiales et sociales de la } \\
\text { clientèle }\end{array}$ \\
\hline
\end{tabular}

\subsection{City logistics : des compétences distinctives pour le grossiste ?}

La logistique, une compétence clé du grossiste ? L'interrogation n'est pas nouvelle et elle interpelle de longue date les milieux professionnels, notamment en termes de gestion des ressources humaines. En effet, parmi les différents métiers logistiques maîtrisés par les grossistes, d'aucuns ont souligné le caractère central de l'expertise liée à la gestion des tournées de livraison, et auxquelles le chauffeur-livreur est directement attaché selon le Rapport d'Activité 2009 de la CGI. Ce chauffeur-livreur a une mission essentielle, celle d'effectuer dans les meilleures conditions de coût et de service les livraisons fixées sur la base d'un planning. Placé à l'interface entre le grossiste et ses clients, il occupe une position charnière : fort de son savoir-faire en matière de livraison des marchandises, il est en mesure de récolter des informations provenant des clients pour les faire remonter ensuite vers les commerciaux. Le Ministère du Travail et des Affaires Sociales (1997) y adjoint une panoplie de compétences complémentaires associées à des activités principales (charger et décharger le véhicule, livrer les clients, contrôler le chargement, rédiger la facture ou le bon de livraison) et à des activités secondaires (préparer les commandes, organiser les livraisons, déterminer l'itinéraire des livraisons).

En bref, les compétences du chauffeur-livreur chez le grossiste sont relatives à des dimensions commerciales, à savoir la connaissance du produit (caractéristiques techniques, prix, évolution de la gamme, etc.) et le contact direct établi avec le client. L'une des explications réside dans le fait que les entreprises du commerce de gros sont souvent de petites structures dans lesquelles, comme pour la plupart des TPE, l'organisation se compose de personnes polyvalentes accomplissant plusieurs tâches (Regnault, 2011). Or, il est possible 
d'envisager de façon simultanée la compétence du chauffeur-livreur dans une perspective de logistique urbaine. C'est en tout cas la conclusion qu'il est possible de tirer des travaux de Cholez (2008). En effet, le chauffeur-livreur cristallise des connaissances spatiales et des connaissances sociales:

- Les connaissances spatiales se traduisent par une fine expertise des secteurs à livrer, telles que les spécificités des rues (les sens uniques, les zones piétonnes, la largeur des voies), qui facilitent ou rendent difficile les arrêts relatifs aux livraisons et le déchargement du camion. En reprenant les travaux de Nonaka et Takeuchi (1995), le chauffeur-livreur dispose à ce niveau un ensemble important de connaissances tacites, pas ou peu formalisées, qui découlent d'une succession d'expériences personnelles ; la transformation de ces connaissances tacites en connaissances formalisées se posera d'ailleurs avec acuité en cas de départ du chauffeur-livreur de l'entreprise. On pourrait penser qu'il s'agit là d'un point purement anecdotique. Or, « dans un environnement urbain dense, l'arrêt du véhicule apparaît toujours comme un moment particulièrement crucial et sensible dans le processus de livraison ou d'enlèvement des marchandises » (Boudouin et Morel, 2002). Une autre connaissance spatiale majeure concerne la réglementation urbaine, parfois spécifique à chaque quartier, comme les espaces sous contrainte d'une durée spécifique de livraison, par exemple de 9 h 30 à 10 h 30, du lundi au vendredi. Le développement des systèmes d'information intelligents, permettant d'orienter n'importe quel chauffeurlivreur, même non expérimenté, en intégrant en temps réel l'actualité du terrain (accidents, bouchons, etc.), pourrait toutefois altérer cette source d'avantage concurrentiel.

- Les connaissances sociales sont relatives aux interactions avec un ensemble d'acteurs de l'espace urbain, les usagers de la voirie d'un côté, les clients destinataires de l'autre. En effet, comme le note Cholez (2008), « les chauffeurs-livreurs doivent respecter les normes de fonctionnement en vigueur (sécurité mais aussi organisation de la production, circulation des personnes, hiérarchies, etc.). Ils doivent également délivrer une prestation dont le contenu est précisément défini lors de l'établissement du contrat de service (porter les colis dans un certain sens, les déposer à un endroit précis) ». Chargement du véhicule et anticipation pendant le déroulement des tournées constituent ici deux compétences clé. L'action de chargement ne se limite pas à charger les colis par ordre de tournées, il «encapsule» des connaissances liées au poids et la taille des colis, ceci afin d'éviter la casse ou la détérioration pendant la livraison. Quant à l'anticipation du chauffeur-livreur, elle se manifeste tout particulièrement en cas d'adaptation de la tournée prévue; en fonction de la fluidité plus ou moins importante de la circulation, le chauffeur-livreur devra faire preuve de réactivité et de prise d'initiative, directement issues de ses savoirs tacites précédemment évoqués.

Bien évidemment, il serait trompeur de réduire l'expertise des entreprises du commerce de gros aux seules compétences de chauffeurs-livreurs ayant assimilé une connaissance « intime » de la ville. Le grossiste est aussi (et surtout) un acteur économique dynamique et innovant ayant su, bien avant les transporteurs messagers, anticiper l'importance des questions d'optimisation logistique dans l'espace urbain (stockage des produits en périphérie, notamment près de zones portuaires et/ou de zones industrielles, ajustement de la capacité des véhicules de livraison aux contraintes spatiales, etc.). La recherche conduite par Cruz (2010) indique ainsi que les caractéristiques du fret urbain, notamment en termes de densité des livraisons, favorisent les grossistes, dont plus de $80 \%$ des établissements sont localisés en ville ou dans sa proche couronne. Inducteur et gestionnaire de flux, le grossiste vit certes sous la menace continuelle de l'intégration de la fonction de gros par ses clients, comme cela est le cas depuis la fin des années 1970. Mais rien ne prouve que les groupes de distribution et les réseaux contractuels ayant investi (ou réinvesti) l'espace urbain puissent faire longtemps l'économie de l'expertise dont il dispose en matière de management de la capillarité, la 
compétence du chauffeur-livreur en étant l'une des facettes singulières. Le grossiste pourra-til cependant avancer seul sur les sentiers escarpés de la logistique urbaine ? C'est peu probable.

Prenons à nouveau l'exemple du TramFret, innovation majeure potentielle s'il en est pour l'acheminement des marchandises en centre ville, qui permet d'éviter les contraintes de circulation dans les zones denses et d'y contourner les réglementations sur l'accès limité des véhicules de livraison. Aujourd'hui, les sociétés de transport de voyageurs possèdent les compétences nécessaires pour acheminer les personnes, mais pas pour acheminer les produits ; contrairement au transport de marchandises, les voyageurs choisissent eux-mêmes le point de départ et le point de destination souhaité, ils prennent individuellement la décision de monter ou de descendre à un arrêt de tramway. En matière de transport de marchandises en ville, le grossiste pourrait ainsi avoir un rôle à jouer en s'associant aux sociétés de transport de voyageurs et proposer une offre globale qui mixerait des compétences complémentaires pour la gestion du dernier kilomètre. Il s'agit là d'une piste de réflexion non explorée pour l'heure, car elle s'appuie sur des partenariats publics-privés techniquement difficiles à mettre en œuvre. Nul doute cependant que la connaissance des contraintes de distribution de la clientèle, pour l'un (le grossiste), la puissance de pénétration capillaire, pour l'autre (l'exploitant du tramway), pourraient trouver à s'allier pour une logistique urbaine efficace et durable.

\section{Conclusion}

Il serait maladroit de considérer que les évolutions contemporaines en matière de logistique urbaine ne concernent qu'une partie réduite des chaînes logistiques multi-acteurs, et qu'elles relèvent uniquement de problématiques techniques liées à l'optimisation sous contrainte du dernier kilomètre. Il est vrai que de nombreux travaux sur la city logistics se cantonnent à cette vision étroite, bien que nécessaire pour le processus de prise de décision (Taniguchi et Thompson, 2008). L'article a indiqué, bien au contraire, que la performance de la logistique urbaine pose avec acuité la question du ou des acteurs disposant des ressources et compétences pour faire face aux enjeux en présence. Une approche stratégique et organisationnelle s'avère par conséquent indispensable, comme elle fut indispensable, il y a deux décennies de cela, pour comprendre et expliquer l'expansion des PSL sur le marché de la grande distribution alimentaire (Fulconis et al., 2011). En effet, gérer des mutualisations horizontales n'est pas simple, des savoir-faire particuliers doivent être mobilisés pour l'occasion, en rupture avec ceux qui fondaient les principes de la massification.

Plus largement, les mutations actuelles de la city logistics interpellent les chercheurs travaillant sur les alliances stratégiques, les coopérations et les réseaux d'affaires. En effet, les politiques de mutualisation, de plus en plus souvent imposées par les collectivités locales, vont conduire des entreprises en concurrence frontale à travailler ensemble, de gré ou de force, sans que ce choix ne découle d'une «stratégie délibérée » de leur part. Accepterontelles une telle contrainte externe sans faire preuve d'une lourde inertie, menaçant de ce fait la réussite des expérimentations en cours? Il serait présomptueux d'adopter trop vite un optimisme béat. Ainsi, dans la principauté de Monaco, alors qu'a été mis en place un important CDU en périphérie de son espace urbain (près de Nice), les magasins Casino continuent à être approvisionnés directement par le système logistique du distributeur (Chanut et Paché, 2012). En bref, le débat reste largement ouvert, il conditionne en grande partie le devenir des nouveaux schémas de la city logistics et, plus largement, de la longue marche vers un monde plus « vert».

Dans une étude comparative désormais célèbre sur l'effondrement de différents systèmes humains (île de Pâques, Mayas du Yucatán, colonies vikings du Groenland, etc.), Diamond 
(2005) a magistralement démontré combien les dommages que les individus infligent consciemment ou inconsciemment à leur environnement participent à un processus de décomposition de Sociétés, parfois très avancées sur le plan économique. Mais il a aussi et surtout souligné les effets funestes de décisions politiques aveuglées par une «volonté de puissance » et un égoïsme de court terme. Loin de sombrer dans un fatalisme sans espoir, Diamond (2005) conclut cependant son essai sur l'effondrement par de multiples pistes de réflexion, en faisant le pari d'un sursaut fondé sur notre capacité collective à tirer bénéfice du savoir accumulé à partir des expériences du passé. Force est d'admettre que les prises de conscience sur l'impératif d'un usage modéré des ressources rares, et le choix de politiques concertées pour éviter les gaspillages, se multiplient depuis dix ans. Si un optimisme béat ne doit certes pas être de mise, ces inflexions en cours, dont les démarches de logistique urbaine mutualisée sont l'une des facettes, témoignent de changements majeurs qui pourraient permettre d'éviter que le syndrome de l'île de Pâques ne se diffuse, pour notre plus grand malheur, à l'échelle planétaire.

\section{Bibliographie}

ADEME (2006), «Transport de marchandises et environnement», Communiqué de Presse du 10 mars, disponible sur http://www.ademe.fr (consulté le 5 décembre 2011).

Augereau, V. (2009), Du transport de marchandises en ville à la logistique urbaine : quels rôles pour un opérateur de transports publics urbains? L'exemple de la RATP, Thèse de doctorat en Transport, Université Paris Est, septembre.

Boudouin, D., et Morel, C. (2002), Logistique urbaine : l'optimisation de la circulation des biens et services en ville, La Documentation Française, Paris.

Cancelier, G. (1981), Le commerce de gros : structures et perspectives, Ministère du Commerce et de l'Artisanat, Paris.

Chanut, O., et Paché, G. (2012), «Stratégies logistiques émergentes : de la grande distribution alimentaire aux réseaux contractuels », Marché et Organisations, $\mathrm{n}^{\circ}$ 15, p. 91-112.

Chanut, O., Capo, C., et Bonet-Fernandez, D. (2011), «De la mutualisation verticale à la mutualisation horizontale: les enjeux et les critères de choix logistiques des réseaux sélectifs contractuels », Revue Française de Gestion Industrielle, vol. 30, n 3, p. 37-59.

Cholez, C. (2008), «Compétences spatiales, compétences d'action dans l'espace: la tournée du chauffeur-livreur », Revue d'Anthropologie des Connaissances, vol. 2, n 1, p. 37-62.

Císařová, H., et Široký, J. (2009), «Logistic technology and the city logistics », Perner's Contacts, vol. $4, n^{\circ} 4$, p. 27-38.

Cruz, C. (2010), « How is own account transport well adapted to urban environments ? », Proceedings of the 12 ${ }^{\text {th }}$ World Conference on Transport Research, Lisbonne, p. 1-19 (CD-rom).

Dablanc, L., Gonzalez-Feliu, J., et Ville, S. (2010), «L'organisation du transport des marchandises en ville : jusqu'où les politiques municipales peuvent-elles juridiquement aller? Le cas de Vicence (Italie) et les leçons à retenir pour les villes européennes », Politiques \& Management Public, vol. 27, $\mathrm{n}^{\circ}$ 4, p. 53-72.

Dagnino, G., et Rocco, E., eds. (2011), Coopetition strategy: theory, experiments and cases, Routledge, Londres.

Danielis, R., Rotaris, L., et Marcucci, E. (2010), «Urban freight policies and distribution channels », European Transport, ${ }^{\circ} 46$, p. 114-146.

Diamond, J. (2005), Collapse: how societies chose to fail or succeed, Viking Penguin, New York (NY).

Drewitz, M. (2007), Transportlogistik mittles Güterstraßenbahn. Einsatzmöglichkeiten im RVR, VDM Verlag Dr. Müller, Sarrebruck.

Dugot, P. (2000), Le commerce de gros, Presses Universitaires de France, Paris. 
Durand, B., et Senkel, M.-P. (2011), «L'innovation au cœur de la logistique urbaine : l'information facteur clé de mutualisation? Étude du cas Cityssimo », Actes de la $2^{e}$ Journée Thématique Systèmes d'Information, Logistique et Innovation, Nantes, p. 1-35.

Filser, M. (1989), Canaux de distribution : description, analyse, gestion, Vuibert, Paris.

Filser, M., des Garets, V., et Paché, G. (2012), La distribution : organisation et stratégie, Éditions Management \& Société, Caen, $2^{\mathrm{e}}$ éd.

Frédouet, C.-H., et Le Mestre, P. (2005), « La construction d'un outil de mesure de la performance des réseaux inter-organisationnels : une étude des réseaux d'acteurs portuaires », Finance Contrôle Stratégie, vol. 8, $\mathrm{n}^{\circ}$ 4, p. 5-32.

Friesz, T., Lee, I., et Lin, C.-C. (2011), «Competition and disruption in a dynamic urban supply chain », Transportation Research Part B: Methodological, vol. 45, n 8, p. 1212-1231.

Fulconis, F., Paché, G., et Roveillo, G. (2011), La prestation logistique: origines, enjeux et perspectives, Éditions Management \& Société, Caen.

Guillo, P.-A. (2008), «Les stratégies d'expansion dans la distribution : le cas des maxidiscompteurs en France », Décisions Marketing, n ${ }^{\circ}$ 50, p. 7-18.

Jeanmougin-Lurdos, C. (1995), La place et la fonction du grossiste dans le canal de distribution : une approche stratégique, Thèse de doctorat en Sciences de Gestion, Université de Bourgogne, janvier.

Juan, S. (2007), «Environnement et écologie urbaine », in Macel, C., et Guillaume, V. (eds.), Airs de Paris, Éditions du Centre Pompidou, Paris, p. 156-160.

Milgrom, P., et Roberts, J. (1990), «Bargaining costs, influence costs, and the organization of economic activity », in Alt, J., et Shepsle, K. (eds.), Perspectives on positive political economy, Cambridge University Press, Cambridge (MA), p. 57-89.

Ministère du Travail et des Affaires Sociales (1997), Le commerce de gros, La Documentation Française, Paris.

Nonaka, I., et Takeuchi, H. (1995), The knowledge-creating company: how Japanese companies create the dynamics of innovation, Oxford University Press, New York (NY).

PIPAME (2009), Logistique et distribution urbaine, Rapport pour le compte du Ministère de l'Économie, de l'Industrie et de l'Emploi, Paris.

Quak, H. (2011), «Urban freight transport : the challenge of sustainability », in Macharis, C., et Melo, S. (eds.), City distribution and urban freight transport: multiple perspectives, Edward Elgar Publishing, Cheltenham, p. 37-56.

de Raymond, A.-B. (2010), «Dispositifs d'intermédiation marchande et politique des marchés : la modernisation du marché des fruits et légumes en France, 1950-1980 », Sociologie du Travail, vol. $52, \mathrm{n}^{\circ} 1, \mathrm{p} .1-20$.

Regnault, G. (2011), Les mondes sociaux des petites et très petites entreprises, L'Harmattan, Paris.

Ripert, C., et Browne, M. (2009), «La démarche exemplaire de Paris pour le transport de marchandises en ville », Les Cahiers Scientifiques du Transport, ${ }^{\circ} 55$, p. 39-62.

Rosenbloom, B., et Larsen Andras, T. (2008), «Wholesalers as global marketers », Journal of Marketing Channels, vol. 15, $\mathrm{n}^{\circ}$ 4, p. 235-252.

Russo, F., et Comi, A. (2011), «Measures for sustainable freight transportation at urban scale: expected goals and tested results in Europe », Journal of Urban Planning Development, vol. 137, $\mathrm{n}^{\circ} 2$, p. $142-152$.

Taniguchi, E., et Thompson, R., eds. (2008), Innovations in city logistics, Nova Science Publishers, New York (NY).

Vandercammen, M., et Jospin-Pernet, N. (2010), La distribution, DeBoeck Université, Bruxelles, $3^{e}$ éd.

Wolf, C., et Seuring, S. (2010), «Environmental impacts as buying criteria for third party logistical services », International Journal of Physical Distribution \& Logistics Management, vol. 40, $\mathrm{n}^{\circ} 1-2$, p. 84-102. 